Revue d'histoire de l'Amérique française

QV REVUE D.HISTOIRE DE L'AMÉRIQUE FRANÇAISE

\title{
L'introduction de la faillite au Bas-Canada : conflit social ou national ?
}

\section{Evelyn Kolish}

Volume 40, numéro 2, automne 1986

URI : https://id.erudit.org/iderudit/304444ar

DOI : https://doi.org/10.7202/304444ar

Aller au sommaire du numéro

\section{Éditeur(s)}

Institut d'histoire de l'Amérique française

\section{ISSN}

0035-2357 (imprimé)

1492-1383 (numérique)

Découvrir la revue

\section{Citer cet article}

Kolish, E. (1986). L'introduction de la faillite au Bas-Canada : conflit social ou national ? Revue d'histoire de l'Amérique française, 40(2), 215-235.

https://doi.org/10.7202/304444ar
Résumé de l'article

Cette analyse des débats sur le droit de la faillite au Bas-Canada essaie d'évaluer l'impact du jeu des intérêts socio-économiques et nationaux ou ethniques sur l'évolution du droit privé dans le contexte colonial. L'auteure constate l'élimination, entre 1760 et 1820, des droits anglais et français sur la faillite par suite de la forte opposition des commerçants au droit métropolitain et de leur préférence pour des procédures plus simples dans les cas d'insolvabilité. Pendant cette phase, la question de la faillite ne suscite que des débats sporadiques, à l'occasion de périodes de crise. De plus, les interlocuteurs sont tous britanniques et les facteurs socio-économiques dominent.

Par contraste, entre 1820 et 1839, le débat s'intensifie et devient presque constant. Le conflit national prend de l'importance lorsque les Patriotes proposent la restauration de la cession de biens. Après une décennie de simple résistance, les marchands britanniques commencent en 1830 à préconiser une réforme selon un modèle plutôt anglais.

L'article examine les techniques juridiques propres à chaque système, français et anglais, ainsi que les conséquences pratiques des diverses options et les divergences entre les groupes en présence. A la lumière de cette analyse, l'auteure conclut que, malgré l'importance incontestable des facteurs socio-économiques dans la naissance d'un mouvement de réforme juridique, le débat sur la faillite ne peut s'expliquer que si l'on tient compte du conflit national.
Tous droits réservés (C) Institut d'histoire de l'Amérique française, 1986

Ce document est protégé par la loi sur le droit d'auteur. L'utilisation des services d'Érudit (y compris la reproduction) est assujettie à sa politique d'utilisation que vous pouvez consulter en ligne.

https://apropos.erudit.org/fr/usagers/politique-dutilisation/ 


\section{L'INTRODUCTION DE LA FAILLITE AU BAS- CANADA: CONFLIT SOCIAL OU NATIONAL? ${ }^{1}$}

EVELYN KOLISH

Montréal

\section{RÉSUMÉ}

Cette analyse des débats sur le droit de la faillite au Bas-Canada essaie d'évaluer l'impact du jeu des intérêts socio-économiques et nationaux ou ethniques sur l'évolution du droit privé dans le contexte colonial. L'auteure constate l'élimination, entre 1760 et 1820, des droits anglais et français sur la faillite par suite de la forte opposition des commerçants au droit métropolitain et de leur préférence pour des procédures plus simples dans les cas d'insolvabilité. Pendant cette phase, la question de la faillite ne suscite que des débats sporadiques, à l'occasion de périodes de crise. De plus, les interlocuteurs sont tous britanniques et les facteurs socio-économiques dominent.

Par contraste, entre 1820 et 1839 , le débat s'intensifie et devient presque constant. Le conflit national prend de l'importance lorsque les Patriotes proposent la restauration de la cession de biens. Après une décennie de simple résistance, les marchands britanniques commencent en 1830 à préconiser une réforme selon un modèle plutôt anglais.

L'article examine les techniques juridiques propres à chaque système, français et anglais, ainsi que les conséquences pratiques des diverses options et les divergences entre les groupes en présence. A la lumière de cette analyse, l'auteure conclut que, malgré l'importance incontestable des facteurs socio-économiques dans la naissance d'un mouvement de réforme juridique, le débat sur la faillite ne peut s'expliquer que si l'on tient compte du conflit national.

\section{ABSTRACT}

This analysis of the debate on bankruptcy law in Lower Canada attempts to evaluate the impact of the interaction of socio-economic

1 Ce texte reprend l'un des thèmes de notre thèse de doctorat, en le développant à partir de sources plus riches. Une version moins détaillée en a été présentée au congrès de l'Institut d'histoire de l'Amérique française en octobre 1985. Nous tenons à remercier André Morel et JeanMarie Fecteau pour leurs commentaires sur une première version de ce texte. 
and national or ethnic factors on the evolution of the private law in the colonial context. The article shows how both English and French bankruptcy law were eliminated after 1760 , largely as a result of the determined opposition of colonial merchants to the imperial law and of their preference for simpler procedures in cases of insolvency. From 1760 to 1820 , the problem of bankruptcy only occasionally gave rise to debate, during times of crisis. In this initial phase, all the participants in the debate were British, and socio-economic factors appear dominant.

Between 1820 and 1839, however, the debate becomes intense and almost continuous. Ethnic conflict becomes an important factor when the Patriotes propose the re-introduction of the French procedure of «cession de biens». In 1830, after a decade of simple resistance, British merchants begin to agitate for reform on the model of British bankruptcy law.

Both English and French legal techniques relating to insolvency are examined, as are the practical consequences of choosing one or the other, and the attitudes towards them expressed by various groups during the debates. In the light of this analysis, the author concludes that, in spite of the undeniable importance of socio-economic factors in the emergence of a movement in favor of a bankruptcy law, the debate on this legal reform in Lower Canada cannot be properly understood without taking ethnic conflict into account.

Le déroulement des débats sur le droit de la faillite entre 1760 et 1840 offre un terrain particulièrement intéressant pour l'analyse du rôle des facteurs socio-économiques et nationaux dans l'évolution du droit privé au Bas-Canada. Peu d'historiens contesteraient l'importance pour la réforme juridique des transformations économiques et sociales. Les tensions socio-économiques se manifestent de façon évidente dans l'évolution de certains secteurs du droit comme la faillite qui s'adresse à un groupe social particulier et à une activité économique spécifique. En effet, les procédures de faillite, développées graduellement en Europe avec l'essor du commerce, visaient uniquement les marchands et les commerçants dans leurs activités commerciales. L'analyse des débats sur la réforme du droit de faillite promet donc d'éclairer le jeu des intérêts entre les milieux commerciaux et les autres groupes sociaux et d'illustrer l'importance de la conjoncture économique en tant que catalyseur de demandes de réforme.

$\mathrm{Au}$ Bas-Canada, cependant, les mobiles socio-économiques des partisans et des adversaires de réformes juridiques se compliquent de préjugés et d'objectifs nationaux. Après le rétablissement du droit civil canadien en 1774 par l'Acte de Québec, chacun pouvait faire appel à 
deux modèles juridiques nationaux chaque fois que la question de la modernisation du droit se posait, ce qui a contribué à paralyser presque complètement l'appareil législatif dans le domaine du droit privé ${ }^{2}$. L'analyse des débats sur le droit de faillite promet de nous aider à mieux cerner l'importance relative des intérêts économiques et nationaux dans le ralentissement de la réforme juridique par législation.

L'évolution des débats se divise en deux phases distinctes. Pendant la première phase, qui s'étend de 1764 à 1820, aucun mouvement de réforme d'envergure n'apparait. Le débat est sporadique; tous les interlocuteurs sont britanniques; et les facteurs socio-économiques dominent. C'est pendant la deuxième phase, de 1820 à 1839, que le débat devient intense et presque constant et que le conflit national devient important. Un examen du cheminement et de la nature des débats dans ces deux périodes nous permettra de mieux comprendre pourquoi.

\section{LA PREMIERE PHASE: 1760 A 1820}

\section{A - Le problème se pose}

Dans cette période, la question de la faillite soulève des débats à deux reprises seulement, à l'occasion de périodes de crise et d'instabilité économique. La première fois en 1767, dans les années difficiles immédiatement après la Conquête, un certain Levi Solomons, ruiné dans les guerres indiennes, ne parvient pas à s'entendre avec ses créanciers et consulte le procureur général Masères afin de trouver une solution à son dilemme. Ce dernier affirme que les lois anglaises sur la faillite "were as much introduced into this province as any other parts of the laws of England» et qu'une «commission of bankruptcy» lui apparaît la meilleure solution ${ }^{3}$.

Cette commission à peine instituée par Carleton, les marchands locaux protestent si vivement que le gouverneur revient sur sa décision. Il s'ensuit un court débat public, entre Masères et quelques marchands britanniques. Ce débat ne touche nullement le droit canadien, lequel n'est pas encore en vigueur. Il vise plutôt à déterminer si le droit anglais de la faillite a été introduit dans la province par la Proclamation royale et s'il convient à la réalité coloniale.

2 A ce sujet, voir E. Kolish, Changements dans le droit privé au Québec/Bas-Canada entre 1760-1840: réactions et attitudes des contemporains, thèse de doctorat, Université de Montréal, 1980. Tout en indiquant les quelques changements dans le droit privé effectués pendant cette période (dont plusieurs potentiellement de grande envergure, comme la liberté de tester), cette étude illustre le blocage relatif de la réforme juridique par législation au Bas-Canada. Le degré de modification du droit par la jurisprudence reste à déterminer, à cause de l'absence de rapports judiciaires publiés et de l'inaccessibilité des archives judiciaires jusqu'à tout récemment. L'analyse des dossiers civils viendra sans doute nuancer notre image actuelle de l'évolution du droit et des institutions juridiques.

3 Masères à Walker, 30 décembre 1768, dans W. S. Wallace, ed., The Masères Letters (Toronto, University of Toronto Studies, 1919), 72. 
A ces questions, les marchands locaux répondent «non». Le 31 décembre 1767 dans la Quebec Gazette on attaque l'affirmation de Masères à l'effet que les lois anglaises sur la faillite ont déjà été introduites par la Proclamation royale. Le journal met en relief le problème général des mécanisrnes de transfert d'un système juridique européen à une colonie. Concédant que les «Englishmen carry the Laws with them wheresoever they go», il se hâte cependant de limiter ce principe aux seules lois qui

... are absolutely necessary to answer the Intention of the Englishmen coming here, such as are requisite in their present State, for the Advancement of their and the general Prosperity, such without which they will neither be protected in their Lives, Liberty, or Prosperity. ${ }^{4}$

Pour la Gazette, le Common Law est à la fois nécessaire et suffisant pour la protection des libertés anglaises. De plus, les lois déclaratoires du Common Law et les lois visant spécifiquement le Québec ou plusieurs colonies lui paraissent applicables ici. En revanche, l'ensemble des lois anglaises ne lui semblent guère appropriées en dehors de la Grande-Bretagne. D'ailleurs, en l'absence dans la colonie d'une décision judiciaire sur le transfert du droit anglais de la faillite, le marchand qui s'en réclame risque éventuellement de voir ces arrangements renversés. La Gazette affirme que les lois ne s'appliquent pas automatiquement dans les colonies, citant à l'appui l'opinion d'un ancien procureur général de l'Angleterre contre l'extension du «Statute of Frauds and Perjuries» à la Barbade. Les marchands du Québec en effet s'opposent à l'introduction du droit anglais de la faillite, rappelant que ni l'Écosse ni les colonies américaines ne l'ont adopté. Ils n'en voient donc pas non plus la nécessité au Québec ${ }^{5}$.

\section{$B$ - Le droit de la faillite en Angleterre}

Quel est donc ce droit métropolitain si redouté par les marchands québécois et, par contraste, quel recours leur offre le Common Law dans les cas d'endettement? Les recours fondamentaux pour les créanciers sont les mêmes en Common Law que dans le droit coutumier de la France (et de l'Europe en général), soit la saisie des biens du débiteur ou la contrainte par corps (l'emprisonnement pour dettes) ${ }^{6}$.

Ce dernier recours avait d'ailleurs été adopté en Angleterre comme un moyen de réprimer les fraudes en 1285, alors que l'essor du com-

4 Quebec Gazette, 31 décembre 1767.

Voir l'article de Masères, qui essaie de répondre aux objections soulevées par les marchands, dans la Quebec Gazette, 10 décembre 1767.

Notez qu'un créancier en Angleterre pouvait utiliser la contrainte par corps contre tous ses débiteurs. En France, cependant, elle était limitée, depuis l'ordonnance civile de 1667, aux litiges entre commerçants et: à certaines catégories de débiteurs spéciaux, comme des tuteurs. 
merce rendait les saisies moins efficaces ${ }^{7}$. Subséquemment, la contrainte par corps avait donné lieu à des abus et le sort des débiteurs languissant dans les prisons fut un sujet fréquent de législation.

$\mathrm{Au}$ début, les lois traitaient seulement de la banqueroute, c'est-àdire des débiteurs de mauvaise foi qui essayaient de frauder leurs créanciers. C'est sous le règne de Henry VIII que la première loi visant spécifiquement la banqueroute fut adoptée; elle autorisait certains fonctionnaires à arrêter le banqueroutier, à saisir ses biens et à distribuer son actif entre ses créanciers. Sous le règne d'Elizabeth $\mathrm{I}$, le système fut restreint aux seuls commerçants et le lord Chancelier reçut le pouvoir de nommer, dans chaque cas, des commissaires (commissioners in bankruptcy) chargés de répartir entre les créanciers le prix de la vente des biens du débiteur. En 1706, l'idée de la faillite (du commerçant honnête mais malchanceux) fit son apparition. Le failli qui se dessaisissait de tous ses biens au profit de ses créanciers et qui se conformait exactement à la loi était déchargé pour l'avenir de tous les engagements contractés avant la faillite pourvu que la majorité des créanciers y consentent. Le failli qui remplissait toutes ces conditions recevait un certificat de conformité pour sa protection ultérieure.

William Blackstone a décrit la procédure normale en cas de faillite au milieu du $18 \mathrm{e}$ siècle ${ }^{8}$. D'abord, le ou les créanciers envoyaient une pétition au lord Chancelier. Ce dernier désignait des commissaires qui devaient déterminer s'il y avait eu faillite. Dans ce cas, ils en donnaient avis dans les journaux et organisaient des assemblées de créanciers. A la première assemblée, on choisissait des syndics (assignees), chargés de rassembler et de liquider les biens et les créances du failli dans un délai d'un an et de distribuer un dividende à chaque créancier, proportionnel au montant de sa créance, mais sans égard à sa nature. Le failli était obligé de dévoiler complètement sa situation financière et de remettre aux commissaires tous ses livres de compte et documents pertinents, sous peine de mort. Les commissaires pouvaient même attribuer une allocation au failli.

Tel était le droit de la faillite en Angleterre, droit qui s'appliquait seulement aux commerçants. Aucune loi anglaise ne réglait le cas des débiteurs insolvables jusqu'en 1813, alors que la procédure d'insolvabilité fut établie pour atténuer les rigueurs de la contrainte par corps. D'autres législations verront le jour après 1845 .

\section{$C$ - Le rejet du modèle anglais}

Au Québec, les objections principales des marchands envers la faillite anglaise témoignent de leur insécurité économique. A leur avis,

\footnotetext{
7 Il s'agit de la 31 Edward I, ch. 3.

8 William Blackstone, Commentaries on the Laws of England (London, Dawsons of Pallmall, 1769), 4 volumes. Réédition (Oxford, Clarendon Press, 1966) 2: 480-487.
} 
les débiteurs peuvent frauder leurs créanciers en utilisant la faillite pour se libérer de leurs dettes. Ils craignent de plus une épidémie de faillites qui ébranlerait la confiance des marchands londoniens à leur égard, d'où le risque d'une diminution de crédit. Ils redoutent aussi que les dividendes soient minimes, vu la nécessité de vendre subitement les biens du failli. Finalement, ils réprouvent l'introduction de commissions de faillite dans la province: d'une part, parce qu'elles seraient trop dispendieuses et, d'autre part, parce que leur participation aux assemblées de syndics prendrait trop de leur temps ${ }^{9}$.

Il est difficile d'évaluer la validité de ces objections. Masères ne les croit pas très réalistes. A son avis, une vraie procédure de faillite encouragerait un accès plus large au crédit, car les créanciers métropolitains auraient ainsi plus de chances d'en profiter qu'avec la procédure de saisie et exécution; celle-ci favorise davantage les créanciers locaux, mieux placés pour surveiller l'état des affaires du débiteur. Les autres appréhensions des marchands lui semblent aussi peu fondées. Quant au coût d'une commission de faillite, Masères l'évalue à 50 livres, montant qu'il estime très raisonnable.

Les adversaires de la faillite s'objectent à l'introduction de procédures nouvelles, potentiellement coûteuses, d'autant plus que leur situation économique leur paraît déjà sombre. En témoigne la lettre suivante:

Consider but how small our Profits are upon Goods, the Length of time before the Capital reenters without our receiving any Interest, even for the Time they remain unpaid after due, and on which we have but a small Profit; the Dearness of all Necessaries, the great Losses we have sustained on our Returns of Peltries, the high Exchange between this Place and London, the uncommon Scarcity of Money, of which we can scarcely scramble enough together to make a few small Remittances (that our Creditors mayn't lose all Patience) and palm the Lawyers with, without which they would not carry on the Suits into which we are forced, by the Spirit of Litigiousness which is so happily spread throughout this colony. ${ }^{10}$

Pour l'auteur de cette lettre, la contrainte par corps reste sans contredit la meilleure protection pour les créanciers. Au droit anglais de la faillite, les marchands préfèrent cette procédure, de même que la saisieexécution $^{11}$.

9 Quebec Gazette, 17 décembre 1767.

10 Ibid., 28 janvier 1768.

1 Notez que la contrainte par corps ne faisait pas réellement partie du Common Law, contrairement à ce que les rnarchands semblaient penser, car elle avait été introduite par la loi de 1285. Sur cette question, William Knox fait un commentaire intéressant dans The Justice and Policy of the Late Act of Parliament for Making More Effectual Provision for the Province of Quebec, Asserted and Proved (Londres, J. Wilkie, 1774), 16: «the truth is, our law of arrests are innovations upon our constitution, and the good of trade has superseded the regard our antient laws shewed for the personal liberty of the subject: And, at this day, there is no country under heaven, where the recovery of debt is attended with more circumstances of cruelty, misery, and slavery than in our own, however we may plume ourselves upon the excellency of our civil code.» 
Bien sûr, un usage modéré de ces procédures paraît nécessaire pour maintenir un sain climat commercial. Selon Masères, les marchands règlent souvent des cas d'endettement par des arrangements privés, où le débiteur cède ses biens à ses créanciers. Il souligne cependant que ces arrangements peuvent être contestés, dès lors qu'ils n'obtiennent pas l'assentiment de tous les créanciers. De là découle, à son avis, le besoin d'une procédure de faillite légale ${ }^{12}$.

\section{$D$ - Le droit canadien de la faillite et son abrogation}

Jusqu'en 1774, le débat ne touche pas ke droit canadien et tous les interlocuteurs sont britanniques, mais l'Acte de Québec, qui restaure le droit civil canadien, change la situation. Toutefois, entre 1774 et 1791 , alors que les plaintes contre le droit canadien fusent librement dans la communauté britannique, le droit canadien de la faillite n'est pas attaqué. Le problème de l'endettement suscite bien des critiques: elles visent plutôt la mauvaise administration des lois qui a entraîné l'abandon du droit canadien de la faillite et qui applique un mélange indigeste de droit anglais et de droit français, et de façon bien arbitraire.

Examinons d'abord la nature du droit français et canadien en matière de faillite et d'endettement avant d'analyser les changements apportés par la législature provinciale et par les tribunaux. Cela nous permettra de situer le débat public dans son contexte global.

Le droit français avait hérité du droit romain la procédure de la cession de biens, où le débiteur se présentait en personne devant le juge et pouvait ainsi éviter la saisie par ses créanciers. Un commerçant pouvait aussi demander au roi une lettre de répit, auquel cas il devait remettre un relevé de tous ses biens et de ses dettes, certifié par le juge et déposé au greffe de la juridiction consulaire. La lettre de répit suspendait les poursuites pendant six mois.

Influencé par la procédure de faillite développée en Italie, le droit français adoptait au $17 \mathrm{e}$ siècle les principes de l'égalité des créanciers et de la période dite «suspecte», période dans laquelle les transactions du failli étaient nulles, afin de l'empêcher de frauder ses créanciers dans les jours précédant la déclaration publique de la faillite. En France, la période suspecte était fixée à dix jours. La grande ordonnance de 1673, le Code marchand, ne contenait qu'un seul article portant spécifiquement sur la faillite et elle passait sous silence plusieurs aspects de la

12 Lettre de Masères, 2 avril 1768, APC, MG 21, Addd. Mss. 3915-2, 266. Il va sans dire que les marchands préféraient éviter complètement les recours juridiques. Ce genre de règlement des échecs commerciaux restera un choix important pour les marchands du Bas-Canada avant l'adoption d'une loi sur la faillite; par exemple, il y a une quarantaine de ces «deeds of assignment» à Montréal entre 1820 et 1830: voir Robert Sweeny, Internal Dynamics and International Cycles: Question of the Transition in Montreal, 1821-1828, thèse de doctorat, Université McGill, 1985, 115 , note 19. D'ailleurs, des accords privés entre créanciers et débiteurs restent toujours, dans des pays de tradition juridiques diverses, une voie d'évitement de la procédure de faillite. 
procédure, tels les rôles de la justice et de l'assemblée des créanciers. Cette assemblée pouvait se prononcer en faveur d'un concordat (un accord par lequel les créanciers remettaient au failli une partie de sa dette) ou d'un atermoiement (un délai pour permettre au débiteur de remplir ses engagements). Les décisions prises par les créanciers qui possédaient ensemble les trois-quarts en valeur des créances étaient obligatoires, quoique le reste des créanciers gardait un droit d'opposition. Sans unanimité, il fallait une homologation en justice. Si le concordat comportait remise, alors les créanciers, comme en Angleterre, ne pouvaient plus entreprendre d'action contre le failli. Le 18e siècle a vu plusieurs édits complémentaires à l'ordonnance.

On remarquera toutefois que les colonies ne se conformaient pas nécessairement aux procédures françaises. Le transfert d'un système juridique d'un pays établi dans une colonie éloignée et peu développée présentait de grandes difficultés ${ }^{13}$. Cependant, bien qu'il n'existe encore aucune étude sur le transfert du droit français en Nouvelle-France, les recherches de John A. Dickinson sur la Prévôté de Québec fournissent certains indices: au moins lorsqu'il s'agissait de procédure, les tribunaux canadiens semblent avoir suivi assez rigoureusement les règlements français ${ }^{14}$.

De toute façon, dans la province de Québec, la cession de biens est éliminée par la jurisprudence en moins de 10 ans. A Montréal, la cour cesse d'accorder la cession en 1781, apparemment parce que le Conseil supérieur n'a pas enregistré le Code marchand ${ }^{15}$. A Québec, les juges n'ont jamais accordé la cession de biens, la considérant abrogée par l'ordonnance de 1777 sur la procédure civile ${ }^{16}$.

Une seule disposition de cette ordonnance (l'article 22) suffit à entraîner l'abrogation de la cession de biens: une disposition qui, contrairement au droit canadien, permet l'emprisonnement pour dettes, de non-commerçants endettés envers des marchands pour l'achat de marchandises. Cette extension de la contrainte par corps était-elle simple-

13 Émilien Petit discute quelques-unes de ces difficultés dans son livre intitulé Droit Public, ou Gouvernement des colonies françoises, d'après les loix faites pour ces Pays (Paris, Chez Delalain, 1771), qui traite principalement de la situation dans les Antilles. Le Common Law et le «statute law»n'ont pas été transférés intacts dans les colonies anglaises non plus. Voir par exemple P. S. Reinsch, «The English Common Law in the Early American Colonies», et St.George Leakin Sioussat, «The Theory of the Extension of English Statutes to the Plantations», dans Select Essays in Anglo-American Legal History, 1: 367-416 et 416-423, Association of American Law Schools (Boston, Little, Brown \& Co., 1907).

${ }_{14}$ J. A. Dickinson, J'ustice et justiciables: la procédure civile à la Prévôté de Québec, 1667-1758 (Québec, Presses de l'Université Laval, coll. «Cahiers de l'Université Laval», no 26, 1982), 74. La description que Dickinson fournit des procès de faillite à la Prévôté (à la page 63) est conforme à la pratique française.

15 J.-F. Perrault, Question et réponses sur le droit civil du Bas-Canada, dédiées aux Étudiants en Droit (s. l., s. é., juin 1810), 4.

16 Voir H. B. Neatby, The Administration of Justice Under the Quebec Act (Minneapolis, University of Minnesota Press, 1937), 158-159. 
ment une mesure de faveur envers les marchands ${ }^{17}$ ou un effort pour les satisfaire, au moins partiellement, en leur donnant un recours juridique qu'ils préconisaient et dont le droit canadien les avait privés? Les deux facteurs ont probablement joué. Cette dernière interprétation semble d'autant plus probable qu'en 1785, alors que l'usage abusif des saisies par les juges du «French Party» avait suscité des plaintes, le Conseil a renouvelé la disposition sur l'emprisonnement pour dettes, la renforçant même par l'addition de la phrase «any law, usage or custom to the contrary notwithstanding».

Les saisies étaient des procédures hybrides, inspirées de façon générale du droit français, mal définies, puis insérées dans la forme de procédure anglaise du capias (le mandat qui ordonnait l'emprisonnement du débiteur ou la saisie de ses biens). Plus librement accordées par la cour de Montréal que par celle de Québec, les saisies étaient appliquées de façon arbitraire et inconstante: à preuve, le célèbre litige entre le gouverneur Haldimand et un certain Cochrane, l'agent de la compagnie chargée de fournir les sommes nécessaires à l'effort militaire. Dans la situation précaire de l'après-guerre, l'affaire Haldimand contre Cochrane avait précipité une vague d'échecs commerciaux et les marchands se plaignaient amèrement de l'indifférence de l'administration de la justice à leur égard ${ }^{18}$.

Cependant, si les marchands en tant que débiteurs redoutent le mauvais usage des saisies, comme créanciers ils ne les détestent pas. En témoigne l'éloge de «X. Y. Z.» à propos de la saisie conservatoire, «one of the best laws which has or can exist in this province» ${ }^{19}$. En l'absence d'une loi sur la faillite, les saisies deviennent essentielles dans une colonie où la plupart des biens des marchands sont des biens meubles, dont les amis du débiteur peuvent facilement disposer pendant son séjour en prison. Comme Neatby conclut: «Carefully defined by exact legislation, and used with moderation and judgment, the saisies might have worked well.» ${ }^{20}$

Les saisies n'ont pas bien fonctionné, non pas à cause de la nature du droit canadien, mais plutôt à cause de la confusion générale dans l'administration de la justice. L'analyse de Neatby présente deux causes majeures de cette incertitude juridique: l'Acte de Québec avait restauré

17 En effet, l'ordonnance mettait les marchands du Bas-Canada dans une position particulièrement privilégiée. Ils pouvaient faire emprisonner leurs débiteurs non commerçants pour dettes pour marchandises, mais n'étaient pas eux-mêmes susceptibles d'emprisonnement pour des dettes envers des non-commerçants. En France, ils n'auraient pas pu utiliser la contrainte par corps contre des non-commerçants et en Angleterre, ils auraient été exposés à l'emprisonnement pour les dettes non commerciales comme tout autre débiteur. Une situation assez cocasse!

18 Pour une description de l'origine confuse des saisies et de leur application arbitraire, voir H. B. Neatby, ibid., 92-101, 160-165, 168-171. Pour l'affaire de Haldimand contre Cochrane, voir 175-195. Pour une description des saisies pendant le Régime français, voir J. A. Dickinson, ibid., $70-73$.

${ }_{19}$ Quebec Herald, 23 février 1789.

20 H. B. Neatby, ibid., 101. 
les lois et coutumes du Canada même si personne dans la colonie ou en Angleterre ne semblait capable d'en déterminer exactement la nature ni de les insérer efficacement dans une structure judiciaire anglaise. Par ailleurs, les juges de la province, à cause de leur manque de formation juridique et de leur engagement politique, n'ont jamais réussi à clarifier la question épineuse de l'étendue précise du droit canadien, ni dans leurs tribunaux ni au Conseil législatif.

Le mécontentement des marchands envers l'usage abusif des saisies ne les amène pas à demander l'introduction de la faillite anglaise. Au contraire, ils restent convaincus que ce droit ne convient pas au pays et préconisent plutôt une ordonnance locale. Ainsi, le rapport des marchands soumis au comité d'enquête sur le commerce en 1787, tout en proclamant le besoin d'une loi sur la faillite pour la colonie, spécifie: «Public credit and punctuality in dealings are not sufficiently established nor in this province ripe enough for the introduction of the Bankrupt laws of England in their full extent...» ${ }^{21}$ Et même un commentateur anglophone suggère le recours au droit français: «an Ordinance should be framed from the Code Marchand.» ${ }^{22}$ Cependant, les conflits politiques de l'époque ne sont guère propices aux réformes juridiques et l'absence d'un véritable droit de la faillite persiste ${ }^{23}$.

\section{E - Une situation juridique tolérable?}

Il faut noter que les plaintes des années 1780 disparaissent après 1791: c'est le résultat de multiples facteurs, entre autres une conjoncture économique favorable aux marchands et le remplacement des juges $\mathrm{du}$ «French Party» par des juristes plus attentifs aux intérêts de la communauté britannique. A l'occasion, un commentaire évoque la difficulté du recouvrement des dettes au Bas-Canada ${ }^{24}$, mais les plaintes ne

21 Adam Shortt et Arthur G. Doughty, Documents Relating to the Constitutional History of Canada, 1791-1818 (Ottawa, 1914), 905-906.

22 Junius, dans le Quebec Herald, 15 mars 1790. Dans la même lettre, Junius suggère aussi de renforcer la procédure de capias, pour mieux protéger les créanciers, en permettant la saisie de biens ou l'emprisonnement des débiteurs pour des dettes moindres que 10 livres et dans tous les litiges, non seulement dans les litiges commerciaux.

${ }_{23}$ Face aux attitudes des marchands envers le droit anglais de la faillite et la suggestion de Junius, pourtant un grand admirateur du droit anglais, de suivre plutôt le Code marchand, l'évaluation suivante de Neatby nous semble plutôt ironique. «The bankrupt laws, or want of them, were one great cause of complaint. Even supposing the code marchand to be in force, that was a strange code, dating for the most part from the preceding century. English bankrupt law had been growing throughout the century, and one of the greatest of English judges, Lord Mansfield was, by his judicial decisions at that time, producing a bankrupt law suited to the needs of a shopkeeping nation.» Neatby, ibid., 47. A la lecture du panégyrique de Neatby à l'endroit du rôle de Lord Mansfield dans la modernisation du droit commercial anglais et du droit de la faillite en particulier, personne ne soupçonnerait que les réalisations de Mansfield «were due largely to the fact that he was widely read in other systems of law than the common law» et qu'il «succeeded in introducing into English law, and adapting to their new environment, some of the principles and rules of continental commercial and maritime law». William Holdsworth, A History of English Law (Londres, Methuen \& Co. Ltd., 1966), 8: 29. 3e édition, 16 volumes.

${ }_{24}$ Voir par exemple Hugh Gray, Letters from Canada (Longman, Hurst, Rees and Orme, 1809), 120. 
sont pas nombreuses et on ne voit guère de suggestions d'une réforme du droit de la faillite avant 1820 .

Le Bas-Canada n'était pas unique dans l'absence d'une loi sur la faillite. Lorsque le Haut-Canada a adopté le droit civil anglais en 1792, il en avait spécifiquement exclu le droit anglais sur la faillite et il n'a adopté aucune loi sur le sujet avant 1840. Les autres colonies de l'Amérique du Nord britannique et plusieurs états américains n'ont pas non plus adopté de législation sur la faillite avant $1850^{25}$.

Il semble raisonnable de conclure que le degré de développement du commerce dans la colonie pendant cette première phase de 1760 à 1820 n'a pas nécessité d'intervention législative. Les moyens juridiques primitifs des saisies et de la contrainte par corps, ainsi que les ententes privées, semblent satisfaire largement les marchands et on discute alors de faillite seulement à l'occasion des crises économiques.

\section{LA DEUXIEME PHASE: 1820 A 1839}

\section{A - Une conjoncture propice au changement?}

Après 1820 cependant, une deuxième phase apparaît: une période de débats et d'activité législative intense sur la question de la faillite. Quels changements suscitent cette transformation? La conjoncture économique semble être un facteur principal. La crise de 1820-1821 et l'augmentation conséquente du nombre de débiteurs suscitent au BasCanada, comme aux États-Unis, des demandes de réforme. On peut probablement trouver dans la croissance économique et démographique de la province l'élément essentiel à l'apparition d'un véritable mouvement de réforme du droit de la faillite. L'économie est plus diversifiée: c'est encore une économie pré-industrielle, mais en transformation, où on voit apparaître, par exemple, les premières institutions financières et un plus grand nombre de sociétés par actions. Les débuts d'une forte immigration britannique augmentent aussi l'élément de risque entre créanciers et débiteurs qui ne se connaissent pas aussi bien qu'ils ne l'auraient pu dans une colonie moins populeuse ${ }^{26}$.

L'endettement semble devenir un problème plus considérable, si on en juge par le nombre de mandats de contrainte par corps émis par la Cour du Banc du Roi de Montréal, lequel augmente depuis 1815. Le

25 Lewis Duncan, The Law and Practice of Bankruptcy in Canada (Toronto, The Carswell Co. Ltd., 1922); Charles Warren, Bankruptcy in United States History, Da Capo Press Reprints in American Constitutional and Legal History, General editor, Leonard W. Levy (New York, Da Capo Press, 1972), 1ère édition (Cambridge, Harvard University Press, 1935).

26 C'est à partir de 1815 que l'immigration britannique «prend un caractère massif et populaire». Les moyennes annuelles d'immigration britannique se situent à 8041 entre 1818 et 1822 , 10867 entre 1822 et 1827,31541 entre 1828 et 1832 et 22444 entre 1833 et 1837 . F. Ouellet, Le Bas-Canada 1791-1840: Changements structuraux et crise (Ottawa, Éditions de l'Université d'Ottawa, coll. «Cahiers d'histoire de l'Université d'Ottawa», no 6, 1976), 218. 
nombre de personnes emprisonnées augmente aussi et le sort des débiteurs qui languissent en prison, dont quelques-uns pendant plusieurs années, suscite l'intérêt du public non commerçant et contribue au mouvement général de réforme qui se dessine ${ }^{27}$.

\section{$B$ - Les initiatives patriotes et la résistance du Conseil et des milieux commerciaux}

Néanmoins, il faut se demander si le mouvement de réforme aurait pu démarrer au Bas-Canada en 1823 sans l'influence du conflit politique et national. Car, curieusement, jusqu'en 1829, les milieux commerciaux ne proposent pas l'introduction d'une procédure de faillite, tandis que les Patriotes la préconisent. Denis-Benjamin Viger présente le premier projet de loi sur la faillite - un projet qui s'alimente du conflit national, justement au moment où le projet d'union de 1822 soulevait de fortes passions. Ainsi, en 1823, Viger soumet à l'Assemblée, pour la première fois, son projet «pour mettre fin à tous doutes relatifs au droit de faire cession de biens», qui propose de renverser la jurisprudence des dernières décennies. Viger et les Patriotes font ici d'une pierre deux coups: ils comblent une carence avec une loi sur la faillite d'inspiration française et condamnent implicitement les juges et conseillers législatifs britanniques qui, à leur avis, avaient «commis la faute d'introduire cette anomalie [la supposée abolition de la cession de biens] dans notre Jurisprudence ${ }^{28}$. Ce projet sera proposé 10 fois entre 1823 et 1836 et adopté 6 fois par la Chambre d'Assemblée, sans jamais réussir à franchir toutes les étapes du cheminement législatif.

La résistance du Conseil et des milieux d'affaires au projet de Viger caractérise la période des débats de 1823 à 1829 , tandis que, entre 1830 et 1839, les demandes de réformes de la part des marchands et d'autres citoyens se multiplient. Apparaissent alors les premiers efforts pour introduire une loi sur la faillite selon le droit anglais.

Pour mieux comprendre le jeu des intérêts socio-économiques et nationaux en présence, examinons d'abord la nature du projet canadien et les motifs de la résistance qu'il a suscitée. Comme le droit anglais de la faillite, la cession de biens ne s'appliquait qu'aux marchands et aux

27 Voir $J C A B C$ (1828), appendice M pour des listes de capias émis à Montréal entre 1794 et 1828 et à Québec entre 1813 et 1828 . Ces listes exigent un traitement systématique par ordinateur que l'auteur effectue actuellement dans le cadre d'une étude sur l'emprisonnement pour dettes au Bas-Canada, à paraître dans La Revue du droit de McGill, 32,4, prévu pour l'été 1987. Un dépouillement préliminaire rapide nous a quand même permis de constater une augmentation considérable du nombre de mandats émis après 1815 (entre 1797 et 1815 , le nombre de mandats ne dépasse pas 50 par année, sauf que 1810-1812 enregistre une hausse à plus de 100 par an; en 1815 , il grimpe à 72 et entre 1816 et 1825 il ne chute jamais en bas de 100 par an). Peut-être cette augmentation tient-elle à des facteurs intangibles, telle une mutation dans les attitudes envers l'emprisonnement pour dettes. Mais il nous semble plus probable que la croissance économique et démographique en soit responsable.

28 La Gazette Canadienne, 13 novembre 1822. 
commerçants et comportait le transfert de tous les biens et revenus du débiteur à une assemblée de ses créanciers ainsi que la révélation complète de son état financier. La cession de biens se distinguait notamment du droit anglais sous deux aspects. Premièrement, le débiteur amorçait la procédure de la cession, généralement pour éviter la contrainte par corps, tandis qu'en Angleterre, les créanciers devaient entamer la procédure de faillite. Deuxièmement, la décharge du débiteur de ses dettes pour l'avenir ne découlait pas automatiquement de la cession. L'assemblée des créanciers pouvait accorder un concordat ou une entente qui comportait une remise ou une décharge, mais ce n'était pas là une conséquence de la procédure de cession de biens. Par contraste, en Angleterre le Parlement avait introduit la décharge dans le droit de la faillite en 1706, sous condition que la majorité des créanciers y consentent. Cependant, le projet de Viger pour rétablir la cession de biens innovait par rapport à la procédure française en proposant que la cession entraîne toujours la décharge, rapprochant ainsi la cession de la procédure anglaise ${ }^{29}$.

Un député canadien, Quesnel, soulève quelques objections au projet, mais la majorité appuie Viger et le projet est adopté. La presse anglophone se divise en deux camps. La Montreal Gazette félicite la Chambre, en rappelant d'ailleurs que la cession de biens existe également en Ecosse. Louant la justice de la loi tant pour le débiteur honnête que pour le créancier, la Gazette conclut:

We are certain, it is the sincere wish of every enlightened mind in the country that Mr. Viger may be successful in his attempt to restore to its full force the cessio bonorum, and that the Province will no longer be disgraced by bankrupt laws at once at variance with the principles of common justice and the dictates of humanity... ${ }^{30}$

L'attitude libérale et approbatrice de la Gazette ne trouve cependant pas d'échos dans le Montreal Herald, l'organe particulier des marchands. Le Herald prend une position très conservatrice, typique du $18 \mathrm{e}$ siècle en Angleterre. A son avis, des lois sévères, appliquées rigoureusement, sont essentielles pour préserver l'ordre social et encourager la probité commerciale. Le journal dénigre les idées des «philosophes modernes» selon lesquelles une trop grande sévérité dans la loi, d'une part, en empêche l'application et, d'autre part, encourage les abus de pouvoir. Il classifie le projet de Viger parmi les «prevaricating, feeble and degrading Laws» inspirées des idées visionnaires dénoncées ${ }^{31}$.

Il semble probable que le contraste entre l'attitude de la Gazette et celle du Herald reflète ici les différences d'opinions auxquelles on s'at-

29 Pour le texte du projet sur la cession de biens, voir Star and Commercial Advertiser, 21 janvier 1829. L'article $X$ du projet aurait établi une décharge automatique.

30 Montreal Gazette, 20 décembre 1823.

31 Montreal Herald, 3 janvier 1824. 
tendrait chez les divers groupes sociaux au sein d'une communauté ethnique assez diversifiée, tant par l'origine de ses membres que par leur appartenance religieuse, leur localisation géographique et leur classe sociale $^{32}$. Il peut s'agir, par exemple, de divergences d'attitudes entre commerçants et artisans ou professionnels ou bien même entre des commerçants intégrés surtout au marché domestique et ceux principalement liés au marché impérial. Dans l'état actuel des recherches, cependant, il ne nous est pas possible d'identifier les attitudes de chacun de ces groupes sociaux au sein de la communauté britannique.

Les arguments favorables ou défavorables à la cession de biens se précisent tout au long de la décennie. En 1825, le Herald révèle la crainte précise qui sous-tendait ses fulminations de 1824: le projet de loi élimine la menace de la contrainte par corps, sans prévoir, aux yeux des marchands, d'autres peines pour empêcher les fraudes ${ }^{33}$. Cette interprétation du projet semble pourtant inexacte. Selon les arguments avancés par Viger dans les débats de 1823, le débiteur frauduleux perd son droit à la cession de biens et reste sujet à la contrainte par corps. Les marchands craignent peut-être que la cession de biens ne laisse aux banqueroutiers le temps de fuir avant qu'on puisse faire la preuve de leurs activités frauduleuses. Un moyen de dissuasion contre la fraude leur apparaît particulièrement nécessaire au Bas-Canada, car à leur avis, l'absence de bureaux d'enregistrement facilite beaucoup les fraudes ${ }^{34}$.

Ce désir de rigueur, afin de protéger les intérêts des créanciers, apparaît aussi dans une série d'amendements au projet de Viger proposés par le Conseil législatif en $1826^{35}$. Ces 27 amendements fournissent les seuls indices explicites pour identifier les motifs d'opposition du Conseil. Les clauses longues et fort détaillées de ces amendements semblent viser à maximiser la sécurité des créanciers et à fixer la procédure de la cession de biens dans ses moindres détails. Par exemple, le serment du débiteur, attaché à l'inventaire de ses biens, détaille toutes les possibilités les plus habituelles de fraude, $\mathrm{y}$ inclus les hypothèques tacites ou expresses ${ }^{36}$. D'ailleurs, une autre disposition prévoit que per-

32 Pour une introduction à la diversité de la population anglophone du Bas-Canada, voir Ronald Rudin, The Forgotten Quebecers: A History of English-Speaking Quebec, 1759-1980. L'importance des entrepreneurs oeuvrant dans et pour le marché interne ressort clairement de la thèse de R. Sweeny, Internal dynamics. Par contre, l'impact des divergences d'intérêts entre les entrepreneurs locaux et les commerçants liés au marché impérial sur le débat sur la cession des biens n'est pas clair.

33 Ibid., 16 avril 1825.

34 Voir par exemple la pétition des marchands du Québec, le 22 décembre 1828, JCABC (1828-1829); le discours de Thomas Lee dans le Star and Commercial Advertiser, 17 décembre 1828; le témoignage de William Patton, dans «Minutes des témoignages pris devant le Comité Spécial auquel a été référé le Bill pour le soulagement, pendant un tems (sic) limité, des Débiteurs insolvables», $J C A B C$ (1831-32), app., Minutes des témoignages, le 24 décembre 1831.

35 JCLBC (1826), 122-134.

36 «I have not at any time created any mortgage tacit or express... other than those which in the said Inventory are set forth...», JCLBC (1826), 124. Cette préoccupation à l'égard des hypothèques reflète le mécontentement des marchands envers le droit hypothécaire canadien. Pour 
sonne ne peut bénéficier à nouveau de la cession au cours des cinq années qui suivent la décharge, à moins que les créanciers possédant les trois-quarts des dettes ne signifient leur consentement, ou que les juges ne trouvent «that this person has, since his or her former discharge, endeavoured by industry and frugality to pay all just demands upon him or her» ${ }^{37}$. Le Conseil prévoit aussi que les tribunaux considéreront les faux témoignages ou les faux serments comme «willful and corrupt perjury» et qu'ils puniront les coupables en conséquence. Ainsi, le Conseil semble partager la crainte des marchands quant aux tentatives de fraudes ét il essaie de limiter l'application de la cession de biens aux seuls débiteurs véritablement insolvables et honnêtes.

Peut-être aussi le Conseil se méfie-t-il d'un projet qui rétablirait une ancienne procédure peu ou mal connue et cela sans en décrire les détails, mais en se référant simplement à l'ancien droit. Sans doute la présence des juges Bowen et Kerr au sein du comité du Conseil responsable des amendements a contribué à cette méfiance envers un simple retour aux sources juridiques françaises, car ces deux juges étaient reconnus pour leur antipathie envers le droit canadien. Ils n'avaient probablement pas bien accueilli non plus la critique de la magistrature, implicite dans le projet.

L'importance que les marchands accordent à la contrainte par corps se manifeste encore plus fortement dans leur réaction envers un projet de loi pour abolir l'emprisonnement pour dettes pour les débiteurs noncommerçants en 1829. Ce projet, proposé par Vallières de Saint-Réal comme complément, et non comme substitut au projet sur la cession de biens, reçoit un appui très ferme de la majorité de l'Assemblée. Celleci adopte une résolution exprimant sa conviction que

... l'emprisonnement, par exécution, d'un Débiteur non Marchand ni Commerçant, pour toutes dettes dues aux Marchands ou Commerçants pour marchandises et effets vendus, est contraire au droit commun du Pays et aux règles de la justice, inutile au commerce et extrêmement nuisible aux autres branches de l'industrie... ${ }^{38}$

Mais le projet suscite une opposition déterminée, qui s'exprime à l'Assemblée et dans la presse ${ }^{39}$. A l'Assemblée, Thomas Lee se fait le porte-parole des marchands, rejetant tous les arguments et les exemples cités par Vallières et Viger. Il accuse les membres des professions libérales «professional men, lawyers, physicians, divines and others» ${ }^{40} \mathrm{de}$ vouloir s'avantager injustement par rapport aux commerçants. Lee a sans doute raison de souligner le rôle des intérêts de classe. Pourtant,

plus de détails sur cette question, voir E. Kolish, Changements, ou «Le Conseil législatif et les bureaux d'enregistrement (1836)», Revue d'histoire de l'Amérique française, 35,2 (septembre 1981): 217-230.

37 Ibid., 133

38 JCABC (1828), 9 décembre 1828.

39 JCABC (1828-29), 160; Star and Commercial Advertiser, 17 décembre 1828.

40 Star and Commercial Advertiser, 17 décembre 1828. 
des nuances s'imposent: les professionnels que Lee attaque sont souvent les créanciers des habitants et n'ont pas la possibilité d'utiliser la contrainte par corps contre leurs débiteurs, car l'ordonnance sur la procédure civile de 1777 ne le permet qu'aux marchands ${ }^{41}$. Il est possible que les professionnells de l'Assemblée, qui ont l'habitude de régler leurs comptes avec leurs débiteurs sans la contrainte par corps, soient moins convaincus de sa nécessité. Certainement, leurs «intérêts de classe» sont moins simples que Lee le suggère, et l'objectif du projet est moins de donner une position privilégiée aux professionnels que de l'enlever aux commerçants. Naturellement, les marchands veulent conserver leur position de force, à moins d'avoir l'assurance d'une réforme qui leur serait aussi avantageuse ${ }^{42}$.

\section{C - De la résistance à un mouvement de réforme}

Après 1830, face aux initiatives répétées de l'Assemblée, et dans une conjoncture économique de plus en plus troublée, les milieux d'affaires commencent à militer pour une commission d'enquête sur le droit de la faillite et de l'insolvabilité. De multiples pétitions de la part de marchands, de débiteurs emprisonnés et de citoyens intéressés sont adressées à l'Assemblée chaque année à partir de $1830^{43}$. La mauvaise conjoncture économique, surtout lors de la crise financière internationale en 1833, semble être le principal facteur qui transforme la résistance des marchands en un désir de réforme. D'une part, en période de crise, les marchands deviennent plus fréquemment insolvables. Ils abandonnent alors leur simple opposition des années prospères, alors qu'ils cherchaient surtout à protéger leur position en tant que créanciers devant des consommateurs issus de tous les groupes sociaux. D'autre part, une mauvaise conjoncture économique, en augmentant le nombre ou l'importance des échecs commerciaux, rend intolérables les imperfections des moyens disponibles pour régler les comptes entre créanciers et faillis. D'ailleurs, dans une de leurs pétitions, les marchands de Montréal déplorent justement l'inefficacité des cessions volontaires (voluntary assignments):

$\ldots$ it is but too notorious that these means are inadequate to effectuate the purpose, for the law not giving to the majority of credi-

41 Le faible pourcentage de mandats d'arrestation pour dettes émis contre des débiteurs canadiens-français (moins que $10 \%$ avant 1815 , et autour de $10 \%$ entre 1815 et 1828 , selon les listes dans l'appendice $\mathrm{M}$ du $J C A B C$ de 1828) semblerait supporter l'hypothèse que les créanciers canadiens-français, même les commerçants qui auraient pu utiliser la contrainte par corps, préféraient d'autres moyens de recouvrement des dettes.

$42 \ll .$. the Petitioners... deem any alteration in the existing laws touching Imprisonment for Debt, not only unadvisable but fraught with danger and injustice for commercial men, unless a Bankrupt law for the relief of merchants and traders, such as is recommended by the wisdom of other countries, and a law for the relief of Insolvent Debtors of other callings be simultaneously enacted.» Pétition du «Committee of Trade» de Montréal, le 10 décembre 1828, JCABC (182829), 160.

43 Pour plus de détails sur les pétitions et les projets de loi, voir E. Kolish, Changements, 519-533 et 624-638. 
tors, the right of administering the debtors' estates, and of binding the whole body by their acts, the objects of such assignments are, in most instances, defeated by some obdurate or unreasonable creditor, who, by the pursuance of legal proceedings, postpones a distribution of the assets, and involves the estate and the creditors in great expense. ${ }^{44}$

Le jeu des intérêts économiques et nationaux devient plus complexe, comme l'illustrent les vifs débats des années 1830. Les divergences d'opinion se retrouvent à l'intérieur des groupes sociaux et des groupes ethniques. Britanniques et Canadiens s'accordent pour reconnaître que l'emprisonnement pour dettes devrait céder la place à une procédure plus moderne et moins cruelle. De cette conviction vient l'appui des Canadiens et d'une partie de la population britannique pour la cession de biens, et pour divers projets visant le soulagement des débiteurs emprisonnés ${ }^{45}$.

Au début des années 1830, l'attitude conservatrice des milieux d'affaires commence à se transformer. La plupart des marchands semblent désormais «convaincû[s] de la nécessité de quelques mesures pour régler et définir les droits réciproques des Débiteurs et des Créanciers dans les cas de Banqueroute et d'Insolvabilité» ${ }^{46}$. Ils réclament à plusieurs reprises, une commission d'enquête.

Cependant, la législature n'arrive pas à se mettre d'accord, ni sur une commission d'enquête, ni sur un projet de loi général. Avant 1835, les seuls projets de loi généraux proposés sont des variations de la cession de biens. Certains commentaires dans le Montreal Herald suggèrent que les commerçants seraient prêts à accepter la cession de biens comme procédure en cas d'insolvabilité, laquelle s'appliquerait à tout débiteur honnête ordinaire, mais non aux marchands, pour qui il faudrait une loi sur la faillite plus spécialisée. Le journal précise cependant, que la décharge devrait demeurer une caractéristique exclusive de la faillite, c'est-à-dire qu'elle devrait s'appliquer seulement aux commerçants, car des lois trop favorables aux débiteurs ordinaires entraîneraient un relâchement des moeurs ${ }^{47}$. Au sujet de la décharge, par

44 Montreal Gazette, 2 avril 1833. Il est possible que certains cas célèbres, comme le litige opposant John Molson à John Richardson, au sujet des accusations de fraude dans la cession volontaire de McGillivray, Thain \& Co., aient contribué à la «notoriété» des déficiences des arrangements privés. Ce litige a été rapporté en détail dans les journaux. Voir par exemple, le Canadian Courant, 2 décembre 1829. L'expérience directe, en tant que créanciers ou débiteurs, fut sans doute importante aussi. Par exemple, quelque 3600 individus ou compagnies ont été impliqués dans les 41 cessions volontaires notariées dressées à Montréal entre 1820 et 1830 et répertoriées dans le «Assignment Cross Reference File» du MBHP (voir R. Sweeny, Internal Dynamics, 115 , note 19.

${ }_{45}$ Par exemple, la Montreal Gazette, 24 mars 1831, applaudit un tel projet parrainé par Ogden, et une pétition de divers marchands et habitants de Québec, JCABC (1831-32), 148, appuie un projet de Duval en plus du projet plus général de Viger.

46 Voir la pétition publiée dans la Montreal Gazette, 2 avril 1833, signée par les membres du «Committee of Trade» en plus de 308 autres commerçants.

47 Herald, 4 février 1829. 
ailleurs, des divergences d'opinion se manifestent au sein des deux groupes ethniques. L..-J. Papineau critique la décharge, même pour des commerçants $^{48}$, tandis que C. R. Ogden, le solliciteur général, propose un projet de loi en 1831 qui établirait une procédure de cession comportant la décharge pour des débiteurs insolvables ordinaires ${ }^{49}$.

\section{D - L'impasse}

En 1835, George Vanfelson propose le premier projet de loi sur la faillite, inspiré des lois impériales, expliquant son initiative par la mauvaise conjoncture économique et les besoins du commerce international:

A bill such as this was the more necessary at the present moment, the state of trade being anything but flourishing. The merchants and manufacturers in England were getting more cautious in their advances to merchands and traders of this country, and it was necessary to adopt some legislative measures, by which confidence would be re-established between the British and Canadian merchants. ${ }^{50}$

Le projet de Vanfelson copie certains aspects de la législation anglaise, par exemple une disposition frappant de peine de mort les faillis qui cachent des biens d'une valeur de $£ 20$ ou plus. Cependant, il innove en proposant d'établir un registre des contrats de mariage pour les marchands, une disposition qui vise les possibilités de fraude laissées ouvertes par le droit hypothécaire canadien et par l'absence de bureaux d'enregistrement ${ }^{51}$.

Son projet se bute au sempiternel projet pour rétablir la cession de biens, proposé en 1835 par Berthelot. Pour la première fois, s'affrontent les deux modèles juridiques nationaux, ce qui ne manque pas de faire ressortir les préférences ethniques. La Montreal Gazette applaudit l'inspiration britannique du projet de Vanfelson ${ }^{52}$. La Minerve, par contraste, considère que le projet de Vanfelson est fautif, en partie parce que «calqué sur les lois étrangères au pays». Il serait «une nouvelle source de confusion dans le système de nos lois, que les décisions injustes ont déjà bouleversées». D'ailleurs, on n'en aurait pas besoin si les juges n'avaient pas «sabordé» la cession de biens, qui elle, pourrait «avec très peu de modifications, répondre aux besoins de toutes les parties intéressées», et de plus, aurait la qualité d'être «fondée sur les principes qui servent de base au corps entier du droit français» ${ }^{53}$.

Quebec Gazette, 27 novembre 1835.

Montreal Gazette, 24 mars 1831.

Vindicator, 31 novembre 1835.

Pour le texte du projet, voir la Montreal Gazette, 21 mars 1835.

Montreal Gazette, 21 mars 1835.

La Minerve, 26 novembre 1835. 
Plusieurs députés patriotes attaquent aussi le projet de Vanfelson pour la sévérité excessive de la clause sur la peine de mort pour les fraudeurs. Berthelot reproche à Vanfelson d'essayer d'introduire sournoisement des bureaux d'enregistrement (prévus pour les commerçants par les articles 15 à 17) dans une loi sur la faillite. Par contre, L.-J. Papineau critique certains aspects des deux projets; il s'oppose notamment à l'innovation de la décharge dans la cession de biens. Ironiquement, Berthelot reçoit l'aide inattendue de Vanfelson dans sa défense du principe de la décharge, bien que l'un invoque des raisons humanitaires et l'autre l'effet bénéfique de la décharge sur le commerce ${ }^{54}$. Finalement, les deux projets de loi échouent.

Le bilan des diverses lois touchant l'endettement et sanctionnées entre 1823 et 1836 montre autant de mesures favorables aux créanciers que de dispositions pour soulager les débiteurs honnêtes ${ }^{55}$. Certains Canadiens proposent des lois rigoureuses en faveur des créanciers, et certains Britanniques proposent des projets pro-débiteurs et inversement. Mais ce sont des lois partielles, qui touchent aux détails du droit existant, et dans le cas de projets généraux sur la faillite, la force d'attraction des modèles juridiques nationaux empêche l'adoption de tout projet identifié à l'une des deux traditions juridiques. L'échec des rébellions et la suspension de la constitution de 1791 brise finalement l'impasse et permet l'imposition par le Conseil spécial en 1839, d'une loi sur la faillite calquée sur le droit anglais, semblable au projet de Vanfelson ${ }^{56}$.

\section{CONCLUSION}

Les débats sur la faillite au Bas-Canada illustrent la complexité du mélange de facteurs socio-économiques et nationaux. Dès la Conquête, la faillite suscite de vifs débats. Au 18e siècle, ces débats sont limités: les marchands britanniques s'opposent au transfert du droit anglais de la faillite dans la colonie, ainsi qu'à l'utilisation abusive des saisies par les juges du «French Party». L'économie de la colonie ne semble pas suffisamment développée ni assez complexe pour susciter un mouvement de réforme avant 1820 . La croissance économique et démogra-

54 Quebec Gazette, 27 novembre 1835.

55 Pour plus de détails, voir E. Kolish, Changements, 519-533 et 624-638. Plus d'une douzaine de projets de loi proposent des modifications mineures aux lois existantes, soit pour renforcer la position des créanciers, soit pour soulager temporairement les débiteurs emprisonnés. Six de ces projets sont adoptés et sanctionnés, dont trois sont favorables aux créanciers et les trois autres favorisent les débiteurs emprisonnés.

562 Vict. ch. 36. 
phique est sans doute un facteur important dans l'intensification des débats après 1820 . Cependant, le facteur économique ne suffit pas à expliquer les initiatives législatives des années 1820 et 1830 et le débat qui les entoure. L'Assemblée, en proposant une procédure de faillite qui s'applique aux marchands et qui libère les débiteurs de leurs dettes, prend une position plutôt favorable au commerce. Cette initiative de la part des représentants d'une petite bourgeoisie supposément ancrée dans une société rurale est même un peu surprenante. Par contraste, à la même époque aux États-Unis, les représentants des états agricoles du sud et de l'ouest s'opposent fortement aux projets fédéraux de loi sur la faillite, tandis que les états du nord-est les appuient ${ }^{57}$.

Remarquons que les membres des comités de l'Assemblée qui endossent systématiquement la cession de biens comptent des hommes de fortune, des hommes qui ne sont pas étrangers au commerce et qui sont souvent des créanciers, par exemple, des notables comme Viger et Augustin Cuvillier. Mais leur initiative législative n'est pas issue des revendications des milieux commerciaux ${ }^{58}$. Cette initiative et la préférence des Patriotes pour le modèle juridique français se comprennent mieux dans le contexte du conflit entre les deux élites ethniques pour maîtriser l'appareil de l'État.

De même, l'opposition des marchands britanniques et du Conseil législatif à la cession de biens ne s'explique pas de façon satisfaisante par leurs simples intérêts socio-économiques. On peut attribuer une grande partie de la résistance à la cession de biens des milieux commerciaux britanniques au conservatisme naturel d'un groupe social favorisé par l'état du droit et qui ne se sent pas sérieusement incommodé par les lacunes du système juridique, du moins jusqu'en 1830. Mais dans le contexte des demandes de réforme des années 1830 , on peut difficilement conclure que les différences entre les deux modèles juridiques justifiaient, du point de vue purement économique, le rejet continuel de la cession des biens. Les débats n'appuient guère une telle conclusion, exception faite de l'inquiétude des marchands face à l'absence de bureaux d'enregistrement. Ce rejet procède en partie sans doute du fait que la cession de biens était le choix des adversaires politiques du parti britannique. Cependant, à en juger par les attitudes générales exprimées envers le droit français, les marchands et le Conseil ont aussi

57 Voir Warren, Bankruptcy in U.S. History, 13, 28, 68.

58 Au moins, sans l'appui des commerçants les mieux organisés et les plus puissants, c'està-dire ceux qui dominaient le commerce international. Il est possible que les commerçants dont les affaires se limitaient à la province étaient moins opposés à la cession de biens. Cette différence d'opinion expliquerait partiellement les initiatives de l'Assemblée, car les Canadiens étaient bien présents dans le commerce interne, comme en témoigne, par exemple, la création de diverses banques canadiennes-françaises au 19e siècle. Voir R. Rudin, Banking en français: The French Banks of Quebec, 1835-1925 (Toronto, University of Toronto Press, 1985). Il est difficile d'évaluer en profondeur cette différence d'opinion, car les sources ne permettent pas d'identifier lesquels parmi les marchands appuient ou s'opposent au projet de la cession de biens. 
résisté aux efforts de restaurer la cession de biens parce que, par principe, la modernisation des lois devrait suivre le modèle anglais et non le modèle français. D'ailleurs, le parti britannique ne croyait sans doute pas qu'une loi d'inspiration française rassurerait les investisseurs londoniens. Ainsi, le débat sur la faillite ne devient vraiment compréhensible que si on tient compte du conflit national, qui bloque l'adoption d'une loi sur la faillite jusqu'à l'échec des Rébellions.

La faillite au Bas-Canada: un conflit social ou national? Inextricablement, les deux. 